\title{
SIN UN CUARTO PROPIO: TRAYECTORIAS JUVENILES Y CUIDADO INFANTIL DURANTE EL AISLAMIENTO POR COVID-19 EN MENDOZA (ARGENTINA)
}

\author{
EMILIANA SEGATORE ${ }^{1}$ \\ MARÍA VICTORIA SECA ${ }^{2}$
}

\begin{abstract}
RESUMEN
La situación de pandemia por Covid-19 que comenzó en 2020 se presentó como una crisis sanitaria, social y económica. El confinamiento al espacio de la esfera doméstica tuvo un correlato en la intensificación de las desigualdades de género y puso en la escena pública la importancia de las tareas del cuidado y su inequitativa distribución. En este artículo buscamos comprender las experiencias de las jóvenes que realizaron trabajo de cuidado infantil no remunerado en este nuevo contexto, a través de los testimonios de mujeres jóvenes de la provincia de Mendoza (Argentina). La investigación tiene un enfoque cualitativo, trabajamos desde una perspectiva crítica, que retoma a los cuidados no remunerados desde la economía feminista, buscando aportar a la sociología del cuidado y de las juventudes a partir de un estudio situado.
\end{abstract}

PALABRAS CLAVES: JUVENTUDES, CUIDADO NO REMUNERADO, PANDEMIA.

${ }^{1}$ Licenciada en Sociología, Universidad Nacional de Cuyo, Argentina. Maestranda en Planificación y Política Social en la misma casa de estudios y se encuentra en la etapa final de acreditación de la Especialidad en Gestión Social. Sus temas de investigación se desarrollan desde la economía feminista y la sociología del cuidado. Es docente de nivel medio e integra un equipo de investigación sobre género, trabajo y cuidados. Correo electrónico: emisegatore@gmail.com

${ }^{2}$ Doctora en Ciencias Sociales, Universidad Nacional de Cuyo, Argentina. Especialista en políticas públicas para la igualdad (CLACSO) y Licenciada en Sociología (UNCuyo). Actualmente es becaria postdoctoral del INCIHUSA-CONICET. Sus temas de investigación se desarrollan desde la sociología de las juventudes y los estudios feministas, con foco en las experiencias de acción colectiva. Es docente del nivel superior e integra varios equipos de investigación con temáticas de juventudes y participación política, cultural y estudiantil. Correo electrónico: victoriaseca@gmail.com 


\title{
SEM UM QUARTO PRÓPRIO: TRAJETÓRIAS JUVENIS E \\ CUIDADOS INFANTIS DURANTE O ISOLAMENTO POR COVID-19 EM MENDOZA (ARGENTINA)
}

\begin{abstract}
RESUMO
A situação de pandemia por COVID-19 que começou em 2020 apareceu como uma crise sanitária, social e econômica. O confinamento à esfera doméstica teve correlação com a intensificação das desigualdades de gênero e trouxe para a arena pública a importância das tarefas de cuidado e sua distribuição desigual. Neste artigo procuramos compreender as experiências das jovens que realizaram trabalho de cuidados infantis não remunerados neste novo contexto, através dos testemunhos de mulheres jovens da província de Mendoza (Argentina). A pesquisa tem um enfoque qualitativo, trabalhamos a partir de uma perspectiva crítica, que retoma os cuidados não remunerados da economia feminista, procurando contribuir para a sociologia do cuidado e das juventudes a partir de um estudo situado.
\end{abstract}

PALAVRAS-CHAVE: JUVENTUDES, CUIDADOS NÃO REMUNERADOS, PANDEMIA.

\section{WITHOUT A ROOM OF THEIR OWN: YOUTH TRAJECTORIES AND CHILD CARE DURING COVID-19 LOCKDOWN IN MENDOZA (ARGENTINA)}

\begin{abstract}
The COVID-19 pandemic situation started in 2020 was presented as a sanitary, social, and economic crisis. The lockdown within the domestic sphere correlated with intensified gender inequalities and brought the importance of care work and its unequal distribution to the public eye. By means of the testimonies from young women from the province of Mendoza (Argentina), we aim at understanding the experiences of young women who have performed unpaid child care work under this new context. We worked on this research, carried out under a qualitative approach, from a critical perspective referring to unpaid care work from a feminist economy, in order to contribute to the sociology of care and youths from a situated study.
\end{abstract}

KEYWORDS: YOUTHS, UNPAID CARE WORK, PANDEMIC. 


\section{INTRODUCCIÓN}

La situación de pandemia por el Covid-19 que comenzó en 2020 se presentó como una crisis sanitaria, social y económica; frente a la cual los gobiernos propusieron diferentes respuestas. En Argentina, desde el 20 de marzo, se dispusieron medidas de aislamiento social preventivo y obligatorio (ASPO) que limitaban la posibilidad de circular por los espacios públicos, asistir a las instituciones educativas de todos los niveles y a los lugares de trabajo, con excepciones de los sectores considerados esenciales (de salud, limpieza, producción, distribución y venta de alimentos). Para el mes de mayo las medidas empezaron a flexibilizarse y comenzó la fase de distanciamiento social preventivo y obligatorio (DISPO), que constó de diversas medidas de apertura o cierre de actividades en pos de disminuir la circulación de personas para evitar la propagación del virus. Dicha situación sigue en vigencia hasta agosto de 2021.

Durante el 2020 las instituciones educativas de todos los niveles (inicial, primario, secundario, superior), tanto de gestión pública como privada, tuvieron que adaptar sus estrategias a la enseñanza virtual. En algunos casos se combinaron con metodologías de enseñanza a distancia, a través de la entrega de material bibliográfico (cuadernillos) que los/as estudiantes pudieron retirar en las escuelas. Esta situación dejó en evidencia el desigual acceso a Internet de la población y de los dispositivos adecuados para el trabajo virtual.

Las medidas implicaron una limitación para el uso de los espacios públicos y el cierre de muchas actividades fuera de los hogares. Este confinamiento en la esfera doméstica tuvo un correlato en la intensificación de las desigualdades de género. Desigualdades dadas por la división sexual del trabajo, que demuestran en última instancia las relaciones de subordinación de las mujeres e identidades feminizadas en una sociedad patriarcal y que implican una organización social y política del cuidado en la misma línea. 
Las desigualdades de género se hicieron evidentes por el aumento de las situaciones de violencia y la centralidad que tomó el trabajo de cuidado no remunerado; es en esta última donde enfocamos nuestro interés. Según la encuesta realizada por UNICEF-Argentina (2020), las tareas dentro del hogar con jefatura masculina - fueron realizadas en un $65 \%$ por mujeres durante el ASPO (3\% más que antes de la pandemia) y en los hogares con jefatura femenina, los números son $78 \%$ y $75 \%$, respectivamente. Lo no tan llamativo de las encuestas es que en épocas de aislamiento, la única tarea en que los varones aumentaron su presencia fue en la realización de compras en el exterior de los hogares; una vez más fue el espacio público el reservado para ellos. Como analizan Rodríguez Enríquez, Alonso y Marzonetto (2020), la situación generada por la pandemia tuvo diferentes repercusiones en la vida de las personas y en la organización social:

(...) al interior de los hogares, en este contexto se observa una sobrecarga de tareas domésticas y reproductivas: mantener el aseo y limpieza de la casa (que en crisis sanitaria implica extremar la higienización); cuidar y entretener a los niños y niñas pequeños; cuidar de los adultos mayores, puesto que incluso los que no presentaban dependencia en otras situaciones ahora son la población más vulnerable frente al virus; garantizar la continuación de los procesos educativos de los niños, niñas y adolescentes haciendo las veces de codocente, ya que se ha resuelto que el proceso de enseñanza-aprendizaje continúe de manera virtual. (p. 2)

Frente a esta situación, nos interesa comprender las experiencias juveniles. Tal como se afirma en el informe, según la encuesta realizado por la OIT (2020), hay que poner en relieve el reto multidimensional que plantea la pandemia para los y las jóvenes debido a la interrupción de sus trayectorias educativas, el aumento en la vulnerabilidad de los/as trabajadores jóvenes y la dificultad de acceder a un empleo decente. De las múltiples dimensiones posibles nos centraremos en las experiencias de las mujeres jóvenes que realizaron tareas de cuidado de niños/as en sus hogares. Particularmente, nos interesa conocer esta situación, ya que anteriormente — según los datos de la Encuesta Nacional de Juventudes (2015) - en Argentina, cuatro de cada diez jóvenes en el país tenían 
responsabilidades de cuidado, sobre todo de niños/as, y la tasa de mujeres que las realizaban duplica a la de los varones.

Nos preguntamos qué hay detrás de esos números y esas afirmaciones en torno a la desigual distribución del cuidado. Atendemos a las particularidades de las experiencias de las jóvenes que realizaron trabajo de cuidado no remunerado en el marco de la pandemia, en pos de analizar las singularidades de un problema de carácter estructural como son las desigualdades de género y la crisis social, económica y sanitaria potenciada por la pandemia. Cómo se sintieron las jóvenes, cómo organizaron sus actividades y rutinas en este nuevo contexto. Qué implicancias consideran que tuvo la organización de las tareas del cuidado durante la pandemia en sus proyectos personales. Trabajamos desde una perspectiva crítica, que retoma a los cuidados no remunerados desde la economía feminista, buscando aportar a la sociología del cuidado y de las juventudes a partir de un estudio situado.

La investigación tiene un enfoque cualitativo. Desde una perspectiva interpretativa desarrollamos cuatro entrevistas a mujeres jóvenes de la provincia de Mendoza (Argentina) que realizaron tareas de cuidado de niños/as en sus hogares durante la pandemia. Los relatos de experiencias que se analizan no pretenden constituirse en representativos o unívocos de las formas en que las jóvenes de la provincia de Mendoza perciben, evalúan y nombran el mundo social que habitaron durante la pandemia. Se trata de una contextualización de un conjunto de narrativas producidas en condiciones históricas concretas, como parte de experiencias, subjetividades y prácticas individuales y colectivas que buscan ir más allá de los números que pusieron en el centro de la escena pública las tareas del cuidado durante el ASPO en el 2020. Lo anterior, con el compromiso de producir conocimiento situado y actualizaciones rigurosas sobre la multiplicidad de significados, prácticas y experiencias desplegadas por las mujeres jóvenes en nuestro país. 
En tanto investigadoras, la temática de los cuidados, de la mano de las tareas con las que nos encontramos en la nueva cotidianidad generada por la pandemia y por situaciones familiares, tuvo especial protagonismo desde marzo del 2020 en adelante. Llevó a que transformemos nuestras rutinas, modos de trabajo y proyectos a futuro; a partir de esta experiencia también valoramos los espacios de diálogo y de reflexión constante con nuestras pares. Esta afectación ${ }^{3}$ igualmente forma parte de nuestras motivaciones para conocer las experiencias de las mujeres jóvenes.

El trabajo está estructurado en cuatro momentos. Primeramente, realizamos un acercamiento teórico hacia los conceptos que guían nuestro análisis situado dentro del estudio de las juventudes y la sociología del cuidado. En segundo lugar, presentamos algunos datos que nos permiten caracterizar la situación que atraviesan las juventudes en la actualidad. Luego, a través de las voces de las jóvenes, analizamos sus experiencias durante el ASPO, buscando conocer el impacto que las tareas del cuidado tuvieron sobre sus trayectorias laborales y educativas, además de las afectaciones que se produjeron en sus vivencias cotidianas, emociones y proyectos de vida, para concluir con las reflexiones finales. Con este artículo no esperamos crear un fotografía estática de la situación ni imágenes recortadas de un puzle, sino los matices de las experiencias donde se articulan los procesos personales y las condiciones estructurales, generando líneas de reflexión teóricas que abren nuevas preguntas en torno a las juventudes y, a su vez, nos habiliten a repensar la organización política y social del cuidado y, en el corto plazo, políticas de corresponsabilidad en el cuidado, incluyendo a las masculinidades y el Estado como coprotagonistas.

\footnotetext{
${ }^{3}$ Como propone Florencia Angilletta (2021) en su libro "Zona de promesas: Cinco discusiones fundamentales entre los feminismos y la política" hay que repensar de qué modo está incluido o contenido quien enuncia en aquello que formula, remarca que es importante tratar de incluir nuestras condiciones de enunciación y no situarse como un sujeto disociado de la escena.
} 


\section{CONSIDERACIONES TEÓRICAS PARA EL ESTUDIO DE LAS} JUVENTUDES Y LOS TRABAJOS DE CUIDADO

Este artículo se sitúa dentro de los estudios de juventudes en articulación con el campo de estudio feminista de la sociología del cuidado, en el marco particular de la pandemia por Covid-19 durante el año 2020. Cuando hablamos de juventudes, la postulamos como una categoría situada en un contexto sociohistórico que adopta sentidos particulares si la comprendemos inserta en el mundo social y con un carácter relacional, ya que el sujeto joven está constituido en y por una trama material y simbólica en el marco de correlaciones de fuerza, también materiales y simbólicas, en el seno de formaciones sociales concretas (relaciones de género, de clase, etnia, territorio y generacionales). Además, es una categoría heterogénea que cuestiona las miradas esencialistas que postulan un único modo de ser joven o que determinan atributos a priori. Como analiza Chaves (2005), giran en torno a un gran no: «Es negada (modelo jurídico) o negativizada (modelo represivo), se le niega existencia como sujeto total (en transición, incompleto, ni niño ni adulto) o se negativizan sus prácticas (juventud problema, juventud gris, joven desviado, tribu juvenil, ser rebelde, delincuente)» (p. 9). Por lo tanto entendemos que no existe «un» sujeto joven, sino una multiplicidad de posibilidades de constitución, aparición y presentación de ese sujeto en el mundo social (Vommaro, 2015).

Dentro de los universos juveniles, nos adentramos en el estudio de las experiencias de las mujeres jóvenes. En la sociología argentina, los estudios sobre juventud nacen en los 80 , se desarrollan en los 90 de manera sectorial y se produce un período de consolidación ya en los 2000; sobre todo luego de la creación de la REIJA — Red de investigadores en juventudes de Argentina — en 2004. En el marco de condiciones institucionales de ampliación de temas, perspectivas y equipos de investigación, tal como analizan Chaves, Cortés, Flaster, Galimberti y Speroni (2013), hay una legitimación de «la juventud»y «los jóvenes» como objeto de investigación científica y una visibilización de las 
prácticas juveniles. A partir de allí, se configuran diversas líneas de trabajo que ponen en vinculación las juventudes con otras dimensiones de la vida social. Se presenta la particularidad de que la línea de trabajo, debate e indagación en torno de la dimensión genérica y sexual de las experiencias juveniles es menor en comparación con las demás. Aunque se evidencia un crecimiento a partir del 2015 en un contexto de la visibilidad pública de las jóvenes en la lucha por sus derechos y ciudadanía sexuales. Este menor desarrollo, como analiza Elizalde (2011):

no significa que las ciencias sociales dedicadas a la indagación de las juventudes no hayan elaborado abordajes puntuales sobre los modos en que las diferencias de género y sexualidad intervienen en la producción de distinciones y jerarquías en la vida de los chicos y las chicas. Implica, más bien, que un número no menor de estas aproximaciones se han formulado desde una concepción binaria y taxonómica de las identidades y expresiones de género y del deseo sexual, y/o se han desarrollado a partir de la tácita asunción de un punto de vista androcéntrico como presupuesto epistemológico de partida. (p. 13)

Por ello, en este artículo entendemos que si pensamos las feminidades juveniles hay que reconocer que existen «feminidades de diseño» (Elizalde, 2011: 13) y experiencias por fuera de estas en donde las jóvenes viven y experimentan muy distintos márgenes de autorrepresentación y de agencia pública respecto de sus actuaciones sexogenéricas. No procuramos dar cuenta de lo que el género y las sexualidades son en o para la juventud, sino de lo que estas distinciones críticas producen y configuran. El lugar que detentan hoy las jóvenes en el orden social y de género se vincula con un conjunto complejo de transformaciones culturales, económicas, políticas y normativas más extensas, previas y en curso. Durante el año 2020, en el marco de la pandemia por el Covid19, con las primeras medidas que limitaron la circulación en el espacio público y sostuvieron el aislamiento social preventivo y obligatorio, las cotidianidades de las jóvenes — como de todos los actores sociales - se vieron modificadas. Particularmente nos interesa analizar sus vivencias en torno a las tareas del cuidado de infancias en sus hogares, pero antes de continuar nos detendremos a 
explicar de qué hablamos cuando nombramos los trabajos no-remunerados de cuidado.

Para nuestro análisis partimos de la premisa de que la desigual distribución del trabajo de cuidado no remunerado, en el que mujeres y disidencias somos las más afectadas en detrimento de la responsabilidad que cabe a varones y Estado, que se traduce, desde hace un tiempo, en una crisis del mismo. A factores como la salida al mercado laboral de las mujeres, la escasa oferta pública de cuidados para la primera infancia y el envejecimiento poblacional, se le sumó la decisión política de aislamiento social preventivo y obligatorio (ASPO), que en nuestro país incluyó el cierre total de los espacios de cuidado y el pase a la virtualidad del sistema educativo, transformando a los hogares en el único espacio posible para niños y niñas.

Los cuidados indirectos refieren a las tareas de limpieza del hogar, preparación de alimentos, compras de insumos, organización horaria y los directos, a la asistencia a otras personas y a sí mismo (Faur, 2014); en su conjunto revisten un carácter de integralidad. Tal como se expresa en la conceptualización realizada por la economista feminista Rodríguez Enríquez (2018), afirmamos que:

(...) cuidado se ha asociado a las actividades indispensables para satisfacer las necesidades básicas de la existencia y reproducción de las personas, brindándoles los elementos físicos y simbólicos que les permiten vivir en sociedad. Incluye el autocuidado, el cuidado directo y de otras personas (...), la provisión de las precondiciones en que se realiza el cuidado (...) y la gestión del cuidado (...). Desde esta visión, el cuidado permite atender las necesidades de las personas dependientes, por su edad o por sus condiciones/capacidades (...) y también de las personas que podrían auto-proveerse dicho cuidado. (p. 134)

En el enfoque macrosocial, el cuidado se trata de una economía mixta, en la que participan diferentes actores: el Estado, el mercado, la familia y las organizaciones de la sociedad civil. Razavi (2007) propone el esquema analítico 
del «diamante de cuidados», cuyas distintas caras —Estado, unidades domésticas, mercado y organizaciones sin fines de lucro- permiten visualizar las instancias proveedoras de los mismos. Como sostiene Faur (2009), en sociedades altamente desiguales como la argentina, no resulta adecuado hablar de un diamante de cuidado, sino que se pueden identificar una variedad de diamantes, definidos principalmente según los niveles de estratificación y fragmentación social, que se reproducen mediante la oferta segmentada de políticas y de diversa calidad según las clases sociales.

En este punto es importante retomar la noción de organización social y política del cuidado como la «configuración que surge del cruce entre la disponibilidad de instituciones que regulan y proveen servicios de cuidado y los modos en que los hogares de distintos niveles socioeconómicos y sus miembros acceden, o no, a ellos» (Faur, 2014: 26). Esta organización, cuando se trata del cuidado de niños/as, históricamente tuvo una impronta maternalista que «intervino en la forma de pensar la maternidad, la paternidad y la crianza, pero también contribuyó a la escasa disponibilidad de alternativas institucionales que facilitaran la re-distribución de los cuidados» (Faur y Pereyra, 2018: 499). Este concepto es clave para comprender la injusta distribución de los cuidados entre los actores definidos en el párrafo anterior y evidenciar que esta desigualdad no es azarosa, sino que es producto de otros factores como la división sexual del trabajo, la naturalización de estereotipos genéricos y el recorrido de las políticas públicas.

La división sexual del trabajo, según Pautassi y Rodríguez Enríquez (como se citó en Rodríguez Enríquez, 2018), «representa la expresión de las relaciones de género en el mundo del trabajo y por tanto muestra relaciones de subordinación de las mujeres» (p. 135). Como expone Pautassi (2019), a pesar de que existan compromisos desde el Estado con organismos internacionales para fomentar la igualdad entre los géneros, a partir del reconocimiento y la valoración de las tareas de cuidado no remunerado y que el mismo se traduzca en servicios 
públicos, infraestructuras y políticas de protección social, esto no resuelve el problema de manera integral. Al mismo tiempo, estas metas incumplidas no se vinculan con las trayectorias laborales de las mujeres, que se ven sumamente afectadas. En este marco de imposibilidades, las más afectadas son las jóvenes pertenecientes a los quintiles de ingresos más bajos, «el tiempo que ellas dedican al trabajo no remunerado y al cuidado de otras personas en el hogar, que representa el doble de tiempo del que destinan los varones, y que repercute en su propia trayectoria educativa, laboral y de vida» (Pautassi, 2019: 145).

En la situación de ASPO, desde el gobierno argentino se tomaron una serie de decisiones que regularon y en menor medida apoyaron a las familias en la responsabilidad total del cuidado infantil, que había recaído sobre ellas a partir del cierre de los espacios educativos y de cuidado. Las mismas incluyeron ${ }^{4}$ : en caso de padre y madre separados se habilitó una autorización de circulación, por única vez, para que el/la niño/a fuera trasladado al hogar del referente principal o del que no fuera personal esencial; en el caso de necesitar de cuidado remunerado, se decretó como personal esencial a las trabajadoras de casas particulares registradas en categoría 4, que corresponde a tareas de cuidado no terapéuticos; en el ámbito educativo se habilitó en la televisión pública programas educativos según nivel educativo y proporcionó material impreso a les estudiantes sin conexión. Como política de transferencia se proporcionó el Ingreso Familiar de Emergencia (IFE) y la ampliación de la «Tarjeta Alimentar» (una política complementaria que buscó garantizar el acceso a la canasta básica alimentaria), la que, en el caso del IFE, fue automática para las familias que poseían la asignación universal por hijo (AUH) y las trabajadoras de casas particulares. A la vez que para los/as trabajadores/as registrados se decretó la posibilidad de acceder a una licencia para el cuidado de sus hijos/as, que permitía

\footnotetext{
${ }^{4}$ Información basada a partir del documento generado en el «Segundo encuentro de la mesa interministerial de Políticas de cuidado», que depende del Ministerio de las Mujeres, Género y Diversidad y que fue realizado en marzo de 2020. Disponible en: https://www.argentina.gob.ar/sites/default/files/politicascuidadocovid19.pdf
} 
la realización de trabajo remoto desde el hogar. En el plano de lo simbólico se realizaron campañas publicitarias sobre la corresponsabilidad en las tareas de cuidado y la incorporación del tema a los contenidos de la educación sexual integral $^{5}$ en las instituciones educativas.

Entonces, qué datos conocemos sobre los trabajos del cuidado no remunerado durante la pandemia de Covid-19 y cómo esto impacta a las mujeres jóvenes, lo desarrollamos a continuación sobre la base de los incipientes trabajos que se han ido produciendo al calor de este fenómeno social que aún estamos experimentando.

\section{ESTADO DE SITUACIÓN: JÓVENES Y CUIDADOS EN EL}

\section{CONTEXTO DE PANDEMIA}

En el escenario latinoamericano prepandemia, a pesar de la mejora de algunos indicadores sociales durante el período 2003-2015, ser joven marcaba un clivaje de la desigualdad:

La reducción de la pobreza, la indigencia y la desigualdad en la distribución del ingreso que ha experimentado la mayor parte de los países de la región no ha permitido, empero, acortar otras brechas que históricamente han definido algunos de los perfiles más notorios de la región. Así, las asimetrías con relación al ingreso y, por ende, al riesgo de caer en situación de pobreza entre grupos étnicos, áreas geográficas (el mundo rural versus el urbano), grupos de edad y género se mantienen desafiantes en la región. (Caetano y de Armas, 2015: 6)

\footnotetext{
${ }^{5}$ En el año 2006 se sancionó en la Argentina la Ley de Educación Sexual Integral, que establece que todos los/as educandos/as de los distintos niveles del sistema educativo tienen derecho a recibir educación sexual integral en los establecimientos educativos públicos, de gestión estatal y privada de jurisdicción nacional, provincial y local. Existen numerosos estudios que analizan los alcances de su aplicación y reconocen la existencia de una brecha entre la propuesta normativa y su ejecución en los establecimientos educativos.
} 
Actualmente, vemos cómo la pandemia está teniendo un impacto negativo sobre las trayectorias laborales de las personas jóvenes. Como se analiza en la encuesta «Jóvenes ante el Covid-19 en América Latina y el Caribe» (Grupo de las Naciones Unidas para el Desarrollo Sostenible, 2020), casi uno de tres jóvenes que trabajan reporta un empeoramiento en su situación laboral debido a la pandemia. Mientras que para muchas personas jóvenes, particularmente mujeres y aquellos en el tramo etario de 20 a 24 años, vivencian el aumento de la carga de trabajo doméstico y de cuidados no remunerados. El 45\% de los/as encuestados/as reconocen un aumento en su carga de trabajo doméstico y de cuidado no remunerado.

La situación de las juventudes de sectores populares en Argentina prepandemia fue similar a la de la región. Se vivió un proceso de generalización de acceso al nivel primario y secundario que estuvo marcado por desigualdades educativas según estrato socioeconómico (Salvia, 2008), tanto por las características de las instituciones educativas como por las desigualdades a la hora de ingresar y permanecer en el sistema educativo. En relación con el trabajo, se vio una mejora de los indicadores de empleo en la Argentina de la posconvertibilidad $^{6}$ que alcanzó al sector juvenil, pero en menor medida que los adultos. La tasa de desempleo fue mayor que la del sector adulto, como así también la precariedad de los puestos laborales (Pérez y Busso, 2018). Según datos de la primera encuesta nacional de juventudes del año 2014, el 53,4\% de

\footnotetext{
${ }^{6}$ El modelo de acumulación que denominamos posconvertibilidad abarca los años del 2003 al 2015, años en los que el país fue presidido por el mismo signo político: Néstor Kirchner (2003-2007) y Cristina Fernández de Kirchner (2007-2011, 2011-2015). En lo económico, frente a la crisis, se consolidó la salida exportadora como uno de los vectores organizadores y dinamizadores de un modelo de acumulación con inclusión social (Manzanelli y Schorr, 2012). En el plano político, se produjo una modificación del rol del Estado en comparación con el período neoliberal anterior, ya que ganó protagonismo, densidad y capacidad de intervención (Carmona, 2015). Como afirma Féliz (2015), «el nuevo desarrollismo vuelve a ubicar al Estado como agente fundamental en su promoción» (p. 103).
} 
los/as jóvenes de ingresos bajos estudiaba, el $46,3 \%$ trabajaba o buscaba trabajo y el $43 \%$ realizaba tareas del cuidado (en su mayoría mujeres).

Casi cuatro de cada diez jóvenes en el país tiene responsabilidades de cuidado, sobre todo de niños (el 34\% de los jóvenes cuida niños habitualmente, dentro o fuera de su propio hogar y un $3 \%$ cuida a adultos mayores). Sin embargo, este dato oculta una gran diferencia de género: la tasa de mujeres que realizan esta importante función social duplica a la de los varones; mientras el $24 \%$ de los varones jóvenes realizan actividades de cuidado, este porcentaje aumenta a $48 \%$ en el caso de las mujeres (De León, 2017: 12).

En el contexto actual, la pandemia y las medidas tomadas para su gestión por parte de los gobiernos nacionales y provinciales no afectaron a todos los grupos sociales por igual. Las consecuencias se produjeron de forma diferencial según clase social, grupo de edad, ubicación geográfica, condiciones de vivienda, acceso a servicios y demás variables que determinan e inciden en todos los fenómenos sociales. A tres meses de decretada la pandemia en Argentina, los datos oficiales mostraron que durante el segundo semestre de 2020 la pobreza $^{7}$ alcanzó al $42 \%$ de la población, afectando en mayor medida a infancias y juventudes: casi seis de cada diez niños/as (de 0 a 14 años) y cinco de cada diez jóvenes (de 15 a 29 años) se encontraron en la pobreza. La crisis está afectando más a quienes tenían una situación más precaria. En este escenario de caída general de la tasa de actividad y del empleo, el sector joven se vio más afectado, principalmente las mujeres. La brecha en la tasa de desocupación entre hombres

\footnotetext{
${ }^{7}$ «En Argentina la pobreza venía en aumento desde 2018. Al segundo semestre de 2020 se observa un incremento significativo de 6,5 puntos porcentuales respecto al año anterior. La recesión global, sumada al cese de actividades por medidas de prevención sanitarias en el contexto de la pandemia, agravaron la crisis que el país ya atravesaba. El aumento que vemos en la pobreza en 2020 se explica principalmente por la caída de los ingresos laborales que los hogares percibieron durante el año. Esto se debió tanto a la pérdida de empleos como a la disminución del poder adquisitivo de sus ingresos en términos reales» (Díaz Lagou, G. y Della Paolera, C., 2021).
} 
y mujeres jóvenes alcanzó un valor crítico de 5,8 puntos porcentuales (INDEC, 2020). Y fueron ellas quienes asumieron también las cargas de las tareas del cuidado al interior de los hogares, lo que sin dejar de reconocer la gran heterogeneidad de situaciones posibles — por estrato social, composición familiar o territorio-, nos permite sostener que «una proporción significativa de mujeres jóvenes y adultas está atravesando la situación actual sin trabajo remunerado, con mayor carga doméstica y con una fuerte dependencia de las políticas estatales alimentarias y de ingresos» (CEPAL, 2020: 24).

\section{MUJERES JÓVENES QUE CUIDAN}

El acercamiento a la temática de estudio fue a través de la metodología cualitativa con entrevistas no $\operatorname{sexistas}^{8}$ - lo que implica que las preguntas no reproduzcan patrones sexistas (cargados de estereotipos) ni miradas androcéntricas - a mujeres jóvenes de la provincia de Mendoza, Argentina, que durante el aislamiento social preventivo y obligatorio en el año 2020 tuvieron responsabilidades de cuidado de niños/as en sus hogares. Las mismas fueron realizadas durante los meses de abril y mayo de 2021, a través de medios digitales. Se utilizó una muestra de tipo intencional. Las autoras no tuvimos contacto previo con ellas de modo directo, sino que fueron convocadas por intermedio de otras personas. Dos, a través de las instituciones educativas a las que concurrían; una, a través de su espacio de participación y otra por su condición laboral autónoma (no registrada). Las cuatro jóvenes habitan diferentes

\footnotetext{
${ }^{8}$ Las investigaciones no sexistas exigen que las relaciones de género se hagan visibles; para ello es preciso tener en cuenta a las mujeres, sus experiencias y su diversidad, así como considerar el papel de las relaciones de género en cualquier análisis social que se vaya a realizar (Díaz Martínez y Dema Moreno, 2013: 69).
} 
regiones de la provincia de Mendoza, todas caracterizadas como zonas urbanas y con ello buscamos no centrarnos en la región del Gran Mendoza, donde se focalizan muchos de los estudios. Esta heterogeneidad de situaciones abonan a la conformación de una muestra rica en experiencias para este trabajo, que busca ser una primera aproximación al universo de experiencias juveniles. El análisis de los datos es de carácter explicativo/interpretativo (Tesch, 1990). Se hizo una lectura pormenorizada de las entrevistas, se procedió a la organización de los datos, su codificación temática y a su integración (Perez, Perez y Seca, 2020), con el fin de lograr una explicación integrada en diálogo con los desarrollos teóricos existentes. No buscamos que los resultados de este trabajo sean extensibles a la situación de todas las jóvenes; nos interesa conocer las particularidades para comprender las múltiples experiencias y modos de transitar los cuidados infantiles durante la pandemia.

Viviana tiene 19 años, es estudiante de nivel secundario en la modalidad de jóvenes y adultos, trabaja en el cuidado remunerado de adultos/as mayores y durante el período de análisis, además, trabajó en una pizzería. Vive en un barrio popular de Godoy Cruz (departamento del Gran Mendoza) junto con su mamá, su hermano pequeño, su hermana mayor y su hija y dos sobrinos de otra hermana; quien no vive en el país. Gleydi tiene 24 años, es estudiante de la tecnicatura superior en enfermería profesional (en un instituto de nivel superior no universitario público), ha tenido varios trabajos precarios y durante el período de análisis comenzó a trabajar en la limpieza de un local comercial. Vive en un barrio popular de la ciudad de Tunuyán (en la región del Valle de Uco de la provincia de Mendoza) junto con su pareja y su hijo de 1 año y 7 meses. Agustina tiene 26 años, es estudiante del primer año del profesorado en lengua y literatura (en un instituto de nivel superior no universitario público), durante el año 2020 no realizó estudios formales y trabajó hasta el mes de julio en un empleo registrado en un call center, luego renunció y concentró sus actividades en torno a su militancia en la campaña nacional por el derecho al aborto. Vive en la ciudad 
de San Rafael (en la región sur de la provincia) junto a su hijo de 5 años. Valentina tiene 21 años, durante el año 2021 comenzó a estudiar el profesorado de educación física (en un instituto superior no-universitario público), en el año 2020 no realizó estudios formales y trabajó de forma autónoma en un trabajo no registrado. Vive en la ciudad de San Rafael junto con su hija de 5 años, su madre y su padrastro. La entrevista estuvo estructurada en torno a tres ejes: las tareas de cuidado que realizaron prepandemia y durante el ASPO, la afectación de las medidas en sus trayectorias personales (con foco en la educación, el trabajo, el tiempo libre) y, finalmente, los aspectos emocionales en torno a sus experiencias. Ejes sobre los cuales desarrollamos el análisis a continuación.

\subsection{EL CUIDADO INTEGRAL EN CONTEXTO DE PANDEMIA}

Las cuatro jóvenes entrevistadas, durante el ASPO llevaron adelante tareas de cuidado directo e indirecto, garantizando la alimentación, vestimenta, apoyo escolar, juegos y el cuidado de la salud de los/as niños/as a su cargo, además de la limpieza del hogar. Un aspecto relevante que emerge de los testimonios es la falta de cuestionamiento hacia las tareas de cuidado. Como explica Batthyány (2013), este cuidado, al darse en el marco de la familia, adquiere un carácter obligatorio pero a la vez es percibido como desinteresado, lo que le otorga una dimensión moral y emocional. A partir del recorrido teórico expuesto con anterioridad, no podemos obviar el hecho de que existen mandatos construidos en las sociedades heterocispatriarcales, que sostienen su distribución desigual al interior de los hogares. Desigualdad que a lo largo del análisis veremos que afecta a las mujeres jóvenes en sus trayectorias y sentires, generando pobreza monetaria y de tiempo, influyendo en su autonomía y que, en última instancia, se reproducen a nivel macrosocial.

Gleydi y Agustina gestionan de manera integral el cuidado, si bien Gleydi convive con su pareja es ella quien organiza las tareas. Por su parte, Valentina y 
Viviana conviven con sus madres y aquí desempeñan un rol más de asistentes que de organizadoras de las tareas del hogar, aunque Valentina gestiona el cuidado de su hija. Esta situación diferente se evidencia en los discursos, a través de afirmaciones como:

Cuido niños, compro los alimentos, hago la comida, ayudo en la limpieza. Las realizo con mi hermana mayor o con mi mamá, dependiendo. (Viviana)

Las comparto por ahí con la familia con la que vivo, que serían mi mamá y mi padrastro, que eso es bueno, obviamente me ayuda un montón y me disminuye un poco estas tareas pero, bueno... entre todos tratamos de compartirlas y de que nos sea más ameno a todos. (Valentina)

A mí siempre me tocó hacer todo, nadie más me ayuda dentro de casa, entonces siempre me tocó hacer todo. (Agustina)

Desde que estamos juntos, siempre fue así de levantarnos y limpiar. Cuando estamos juntos lo hacemos los dos. Cuando estoy sola, soy solo yo. (...) él me ayudaba. Él me ayuda muchísimo. (Gleydi)

Los testimonios nos permiten evidenciar no solo el modo en que se distribuyen las tareas, sino sobre quién recae la planificación, gestión y organización del mismo, lo que genera en la responsable una carga mental situación que comparten Agustina y Gleydi-. Esta noción hace referencia al conjunto de tensiones, al esfuerzo mental y el trabajo cognitivo que conllevan las actividades y tareas en el ámbito doméstico, visibiliza el proceso de toma de decisiones que implica el cuidado: organizar qué cosas hay que hacer, cuándo hay que hacerlas, decirles a otras personas lo que tienen que hacer para asegurar que ese cuidado se realice. En términos teóricos vemos coómo en el caso de Gleydi y Agustina se produce una «doppia presenza» (Balbo, 1978) que da cuenta de la doble tarea que realizan las mujeres insertas en el mercado laboral y que a su vez no abandonan las tareas domésticas; esa doble presencia expresa el vínculo sincrónico entre tiempo y trabajo. La afirmación de «él me ayuda muchísimo» pone en evidencia la internalización de los mandatos culturales y los estereotipos hegemónicos de masculinidades — el varón, en tanto proveedor, no 
tiene la responsabilidad de realizar esas labores - y feminidades. Como así también las jóvenes que viven con sus madres y se reconocen como «colaboradoras» en las tareas. Es interesante esta diferencia porque matiza las diversas situaciones que podemos encontrar detrás de los números de aumento del trabajo de cuidado.

Todas ellas coinciden que, durante la pandemia, las tareas de cuidado que realizaban en sus hogares se vieron incrementadas. En parte, por el protagonismo de la limpieza de los ambientes como medida de prevención contra la propagación del Covid-19. También, en el caso de las jóvenes que tenían a niños en edad escolar a cargo de su cuidado, por el cambio de modalidad de cursado presencial a virtual. Esta medida tomada por el gobierno nacional significó un doble perjuicio para ellas: la pérdida de un espacio de cuidado y el aumento de tareas a partir de la realización de actividades escolares, para lo cual no se sentían capacitadas. En lo que respecta a la organización educativa en Argentina, «el diseño de estos servicios — y su evolución — refleja el modo en el cual el Estado entiende las responsabilidades institucionales en la atención de niños» (Faur y Pereyra, 2018: 500), y la escolarización permite a las familias organizar su dinámica contando con ella como espacio de cuidado.

Sí, aumentó mucho porque ya no tenían clases, entonces era todo el tiempo ya cuidarlos, y... casi todo el día porque era cuando mi mamá se iba a trabajar, cuando mi hermana se iba a trabajar, así que era casi todo el día. (Viviana)

Durante la pandemia, además, recae la enseñanza escolar y la verdad que tiene un colegio divino y las maestras son súper divinas. Pero también una tiene que tomarse su tiempo para tratar de ser lo más didáctica posible, cuando yo estoy estudiando un profesorado de secundaria, así que de nivel inicial cero. (Agustina)

Sí puedo llegar a decir que en la cuarentena aumentaron estas tareas porque.... por el hecho de que la niña no podía realizar ninguna actividad fuera de casa y se sumaron las tareas, las clases online, donde, bueno, nada, tiene su dedicación un poquito más... y bueno, pasas más tiempo con ellos, obviamente, en la casa. (Valentina) 
Como afirma Arza (2020), en el informe de la CEPAL:

El cuidado puede ser prestado por miembros de la familia (madre, padre, abuela, etc.) o puede ser «desfamiliarizado», es decir, externalizado y provisto por individuos o instituciones fuera del hogar, tales como personas que no pertenecen a la familia y prestan ayuda solidaria (vecinos/as, amigos/as), trabajadores y trabajadoras remuneradas del cuidado, o instituciones públicas, privadas o comunitarias. (p. 49)

Es interesante ver cómo, con el paso de los meses de pandemia, las jóvenes han desarrollado estrategias de resolución de las tareas y volvieron a gestionar redes de cuidado, según lo expone Pérez Orozco (2007) en Rodríguez Enríquez (2018), como una red de encadenamientos múltiples y no lineales «que se dan entre los actores que participan en el cuidado, los escenarios en los cuales esto sucede, las interrelaciones que establecen entre sí y, en consecuencia, lo densa o débil que resulta la red de cuidados» (p. 133). Lo anterior, con la finalidad de disminuir el peso del trabajo del cuidado de los/as niños/as, ya sea para tener un momento en soledad, para estudiar o para trabajar. En ningún caso esta red pudo ser cubierta más que por la familia, reproduciendo una de las principales características — junto con la feminización — de la organización social y política del cuidado en Argentina.

Armamos una burbuja familiar con los abuelos y mi tía y mi primita más chica, cosa de poder vernos entre nosotras y eso fue muy productivo, pero solo era un día del fin de semana. (Agustina)

Mi marido iba a veces y a veces no a trabajar, porque es militar, entonces para poder ir a trabajar yo le dejaba mi bebé a él o a mi mamá y volvía, y si el bebé seguía durmiendo yo aprovechaba para estudiar. (Gleydi)

En este contexto, la desigual distribución del cuidado entre los actores intervinientes, materializada en la organización social y política del cuidado, llegó al punto máximo de familiarización, a través de la ausencia total de oferta pública de cuidado por parte de los estados municipal, provincial y nacional y la prohibición de la oferta privada por las medidas sanitarias. Es importante tener 
en cuenta que en Argentina, al igual que en la región exceptuando a Uruguay, existen instrumentos en términos de política de cuidado que proveen alguna solución al problema, pero no un sistema integral, lo que quedó en evidencia con la pandemia.

\subsection{LAS MODIFICACIONES EN SUS TRAYECTORIAS VITALES}

Hay un vasto desarrollo de estudios que atienden las trayectorias juveniles en Argentina frente a los cambios sociales. En general, se han caracterizado por combinar elementos macro y microsociológicos y trabajar sobre el par dicotómico inclusión-exclusión, lo que implica que las lecturas se realicen en claves de integración (o no)9 al sistema social dominante. El contexto particular de la pandemia nos lleva a preguntarnos nuevamente sobre las trayectorias personales de las jóvenes, con foco en los aspectos tradicionales de análisis (la educación y el trabajo), al que le sumamos la dimensión del tiempo libre entendido como aquellas actividades que realizaron (o realizan) de manera voluntaria en el tiempo que no destinan a trabajar ni a estudiar, como son las actividades de participación militante, religiosa, cultural y deportiva.

Sin duda las vidas de todos/as se vieron modificadas a partir del avance del Covid-19 y con la definición de la situación como pandemia por parte de la OMS, así como con las medidas que los gobiernos tomaron en consecuencia. En esta oportunidad vemos cómo las trayectorias personales de nuestras entrevistadas cambiaron; el tiempo disponible se convirtió en escaso, muchas

\footnotetext{
${ }^{9}$ La escuela sociológica francesa desarrolló, mediante la obra de Robert Castel, un trabajo teórico de sumo interés para abordar los procesos de integración-desintegración social en las sociedades del presente. Castel (1997) sitúa la problemática de la integración y de la cuestión social en perspectiva histórica y es la base de su análisis sociológico. Su mirada permite delimitar continuidades y rupturas de los procesos analizados, ayuda a identificar las transformaciones históricas, lo permanente y lo nuevo.
} 
actividades fueron restringidas, algunos trabajos no fueron contemplados como esenciales y, por lo tanto, también restringidos.

En el período de análisis que incluye los meses de marzo a junio del año 2020, Gleydi y Viviana estudiaban y ambas tuvieron dificultad para mantener una trayectoria continua debido al aumento de las tareas de cuidado, la complejidad que presentaba de la modalidad virtual y la superposición con el trabajo remunerado.

Me afectó un poco porque era hacer las tareas de ellos y al mismo tiempo trataba de hacer mis tareas y fue como que medio que me iba y... aparte estaba trabajando también, así que era como que no podía dividirme (Viviana)

Durante la pandemia, lo escolar me afectó muchísimo. Porque la institución fue muy desorganizada, que me hubiesen gustado que fuera de otra forma. La modalidad virtual en algunas materias no fue buena, no había buena explicación, a veces no tenían buena conexión los profesores y se les escuchaba todo cortado. Entonces es más lo que perdí. (...) Lo que me pasaba a mí es que a veces el profesor explicaba y explicaba en mi horario de trabajo; entonces ahí se me complicaba. (Gleydi)

Además, presentaron un malestar personal respecto al nivel de aprendizaje y las calificaciones obtenidas: «No sé cómo acredité algunas materias. La verdad me hubiera gustado aprender más», afirma Gleydi. Según ellas, esto se produjo como consecuencia de la imposibilidad de conciliar trabajo remunerado con trabajo no remunerado y estudio, y por la complejidad de estudiar de modo virtual. Situación que, en el caso de Viviana, se vio afectada por los problemas de salud de una de sus sobrinas que pasó tiempo hospitalizada.

Agustina y Valentina comenzaron a estudiar durante el 2021; aunque no es parte del período de nuestro análisis, queremos hacer dos comentarios al respecto. Por un lado, el impasse que tuvo Valentina en su trayectoria desde que finalizó la escuela secundaria e ingresó al nivel superior es explicado por ella debido a la imposibilidad de conciliar estudio y cuidados: «Egresé de la secundaría en 2017, tuve que esperar un poco a que mi hija crezca». Por otro 
lado, ambas reconocen que la modalidad virtual les ofrece mayores facilidades para poder continuar su formación. Como ya se dijo, en Argentina se adaptaron las trayectorias educativas en formato virtual por la pandemia y, en el caso del nivel superior, la metodología de trabajo durante el 2021 continúa del mismo modo. Se estipula que durante los meses de agosto y septiembre se producirá la vuelta a la presencialidad, entonces al momento de entrevistarlas, ellas no se habían problematizado sobre cómo lidiar con la organización de los cuidados de los/as niños/as y el estudio en las instituciones educativas.

A mí, la verdad se me hace mucho más fácil cursar así, desde la virtualidad, porque no tengo que dejar a mi hija, no tengo que buscar a una niñera o a alguien que la cuide, entonces, bueno, en ese sentido se me hace mucho más cómodo, más práctico estar desde casa. (Valentina)

Las cuatro jóvenes están insertas en instituciones educativas que han sido estudiadas como espacios educativos, espacio de interacción social y de construcción identitaria y biográfica (Núñez y Litichever, 2016). Sin embargo ninguna de ellas las referencia de ese modo; posiblemente esto se haya visto limitado al trasladarse el cursado a la virtualidad. A la vez, se evidencia la diferencia entre quienes estaban insertas en las mismas previo a la pandemia y quienes comenzaron sus estudios durante el aislamiento, mostrando valoraciones diferentes, que pueden leerse hasta contrarias.

Respecto al trabajo remunerado, como vimos en el apartado anterior, la participación femenina y las horas dedicadas a él presentan niveles mucho más bajos que en los varones y en mayor medida cuando existen responsabilidades de cuidado. Como expone Rodríguez Enríquez (2005), estas posibilidades u obstáculos que enfrentan las mujeres para participar del trabajo remunerado tiene implicancias en su independencia, realización personal y contribuir al bienestar de su hogar. No fue objeto de nuestro análisis estudiar minuciosamente las trayectorias laborales de las jóvenes, sino comprender cómo se construyeron en el período de estudio. Nos encontramos con una situación heterogénea entre las 
entrevistadas; en parte comprensible por los diferentes capitales económicos, sociales y culturales (Bourdieu, 1997) con los que cuentan cada una de ellas.

En el caso de Valentina, las medidas sanitarias tuvieron como consecuencia la pérdida de su empleo remunerado y autónomo. La actividad de masajes no estuvo permitida durante el ASPO, lo que llevó a que dejara de trabajar y con ello de percibir ingresos (por su carácter de autónomo y no registrado). A esto se le sumó el «miedo» de las personas de ser contagiadas al asistir a una sesión. Viviana era empleada en una casa particular, actividad que no estaba permitida pero que la normativa intimaba a los/as empleadores a continuar pagando el salario, lo que no sucedió en su caso porque ella lo realizaba sin contrato formal. En el país, el trabajo de limpieza en casas particulares tiene un nivel de informalidad de 75\%, por lo que muchas personas (un $98 \%$ de mujeres) dejaron de percibir sus ingresos al comenzar el aislamiento. Ello la llevó a cambiar de actividad y comenzó con dos nuevos trabajos de manera no registrada (cuidado de adultos/as mayores y en una pizzería).

Gleydi también realizó trabajos de limpieza, pero no en casas particulares, sino en un local comercial. Comenzó su actividad en el mes de junio de 2020 cuando, dentro de las medidas de aislamiento se habilitaron las ventas allí, por lo tanto, si bien su trabajo también es precario, tuvo continuidad en el mismo hasta la fecha. Además, ella remarcó que realizó su tarea con todas las medidas de bioseguridad recomendadas — aunque los elementos se los proveía ella misma. La experiencia de Agustina fue diferente. Al comenzar la pandemia tuvo un trabajo registrado que cambió su modalidad de presencial a teletrabajo. Debido al agotamiento que le implicó estar todo el día en su casa — con las múltiples tareas-y por contar con capital económico, eligió dejar su trabajo. Luego de unos meses sin trabajo remunerado, en los que se abocó a sus tareas en tanto militante de la campaña nacional por el derecho al aborto legal, seguro y gratuito, comenzó a realizar una labor periodística en medios de comunicación locales. Estas situaciones particulares nos permiten ver cómo los factores estructurales 
impactan diferencialmente en las trayectorias personales de las jóvenes, y cómo los capitales económicos, sociales y culturales con los que cuenta cada una dejan en evidencia las diferentes situaciones de precariedad vividas.

Al dedicarles más horas a las tareas del cuidado, como vimos, las jóvenes deben organizar su tiempo de estudio y de trabajo remunerado, como así también otorgarles (o no) espacio a las actividades recreativas. Katzkowick et al. (2015) afirman que «las horas de ocio se ven cercenadas al asumir estas tareas» (p. 8). El tiempo de ocio de las jóvenes se redujo considerablemente a partir del confinamiento en sus hogares, sobre todo por no poder asistir a los ámbitos de socialización donde disfrutaban de su tiempo libre ( $\sin$ tareas de cuidado y de trabajo remunerado). Las actividades que hacían eran diferentes: Viviana y Valentina participaban de espacios deportivos; Gleydi asistía a las actividades religiosas de su iglesia; Agustina formaba parte de un espacio de militancia feminista por el derecho al aborto. Por las medidas sanitarias, dichos espacios se transformaron y no todas pudieron reconvertir sus prácticas para generar los espacios de tiempo propio/recreativo.

Antes hacía fútbol y después de la pandemia no pude seguir, ya que nos cancelaron y el equipo se desintegró. (Viviana)

Antes de marzo, la única actividad que puedo hablar que es la que hago es ir a la iglesia (Gleydi)

Tuve que dejar mis actividades, como el entrenamiento. (Valentina)

En junio, julio, agosto, empezamos de nuevo con la movida de militancia (...). Llegó un momento que tenía la casa dada vuelta, llena de banderas y pañuelos y Juani estaba chocho. (Agustina)

En el caso de Agustina, sí pudo reconvertir su espacio de militancia —a través de las actividades virtuales - y conciliarlas con las tareas de cuidado, aunque no logró reemplazar su presencia en las mismas por la adaptación de su hijo. Esa posibilidad de continuar su actividad le provocó satisfacción, a diferencia de las demás que notan la carencia de esos espacios, con sus actividades específicas y el encuentro con otras personas. 


\subsection{LAS EMOCIONES DE LAS JÓVENES EN TIEMPOS DE PANDEMIA}

La emoción solo puede entonces manifestarse empotrada sobre lazos de sociabilidad, ya que es el conocimiento de estos lazos y de estas relaciones el que brinda la posibilidad misma de esa emoción (Daich, Pita y Sirimarco, 2007: 77).

Una tercera dimensión de análisis son las emociones y sentimientos que se pusieron en juego al momento de declararse el ASPO en Argentina. Como ha sido ampliamente estudiado, las emociones tienen un carácter relacional, en tanto constructos sociales y culturales y como significados motivados culturalmente o socialmente articulados (Ahmed, 2008; Daich, 2017; Lutz y White, 1986). Por ello, hablar de emociones implica también hablar de vínculos. Y no implica solo una afirmación sobre el estado interno de los sujetos, sino también una afirmación sobre las relaciones que vinculan a los sujetos entre sí y a estos con distintos eventos, como se expresa en la nota inicial.

Gleydi centró sus referencias en su vínculo de pareja, con quien convive, y también en su madre, al momento de explicar quién la ayudaba con el cuidado de su hijo. Si bien ella cursaba sus estudios superiores en Enfermería, trabajaba fuera de su hogar y asistía, antes de la pandemia, a una iglesia. Sin embargo, no nombra en ningún momento relaciones o vínculos con personas de esos espacios de socialización. Viviana, quien vive con su madre y su hermana, asiste a la escuela y además trabaja de modo informal, y tampoco hace referencia a los vínculos que se pueden llegar a construir en dichos ámbitos de socialización. Sus amigos y amigas, que conforman su red vincular, se han dado en el territorio donde vive, en el barrio. Agustina nombra a su familia — su prima, su abuelo y su abuela, su tía - con quienes realiza reuniones y la ayudan (en la prepandemia y durante) con el cuidado de su hijo. No presenta al ámbito laboral y de estudio como espacios centrales para la construcción de vínculos. Esta centralidad la tiene su espacio de participación política, ya que para ella sus amigas son sus compañeras de militancia feminista por el derecho al aborto. Valentina nos habla 
de los vínculos con su mamá y su padrastro y las relaciones que establece con sus amigas (a quienes conoce por el deporte y su colegio secundario) y entre quienes incluye a su hermana, con quien no convive pero que también es madre joven como ella. Estos vínculos forman parte del entramado relacional en el cual las jóvenes desarrollan su cotidianeidad, gestionan sus emociones y configuran sus expectativas.

Las tareas de cuidado que llevaron adelante son también enmarcadas teóricamente como un trabajo con un marcado carácter emocional, encaminado al bienestar subjetivo de las personas. Cuidar es un trabajo afectivo (Vega Solís, 2009) tanto para las personas que los reciben como para quienes lo administran. Uno de los sentimientos que salió a la luz fue la incertidumbre acerca de lo que podía llegar a pasar en sus casas, con sus seres queridos, en su comunidad tanto en el plano de la salud como en el económico y de organización diaria-. Se vincula con la situación incierta, propia del contexto de pandemia que no permite planificación y que conllevó también una gran preocupación en ellas. Por otro lado, el miedo. Miedo a enfermarse, a que se enferme alguien de su familia y, en el caso de Gleydi, también el miedo a la escasez de alimentos y de trabajo. Este hallazgo va en la misma dirección que los aportes de la investigación realizada por Johnson, Saletti-Cuesta y Tumas (2020)10, quienes destacan que la incertidumbre, el miedo y la angustia son los principales sentimientos generados por la pandemia.

Me sentí un poco mucho colapsada, porque era como que todo se me había venido abajo, pero dentro de todo, lo pude superar. Fueron de frustración, me sentía mal. (Viviana)

\footnotetext{
${ }^{10} \mathrm{El}$ trabajo explora los sentimientos y expectativas que generó el Covid-19 en Argentina durante la primera etapa de la pandemia. Sigue la propuesta del estudio «COVID-19 Snapshot Monitoring (COSMO): Monitoring knowledge, risk perceptions, preventive behaviours, and public trust in the current coronavirus outbreak», elaborado por la Oficina Regional Europea de la OMS y que tomó datos durante fines de marzo y comienzos de abril de 2020 .
} 
La verdad que fue muy estresante mentalmente y tener que estar dispuesta a todo (...). La mayor carga que noté fue emocional. (Agustina)

Tanto Viviana como Agustina comparten un sentimiento de agobio por la interrupción abrupta de la cotidianeidad como la conocían; evidenciado en expresiones como las de colapso y estrés. Posiblemente producto de que la mayor parte de sus interacciones emocionalmente significativas no ocurrían al interior de sus viviendas, sino con sus pares generacionales. Por otro lado, el contexto de pandemia llevó a que desarrollaran nuevas rutinas que no las satisficieron, viéndose condicionadas por la convivencia - con sus hijos o parejas - y los cuidados especiales a personas enfermas de la familia:

Es muy raro el encierro. (...) Porque yo toda mi vida trabajé y era sentarnos y mirarnos a la cara o mirar películas y se volvía aburrido. Me sentía aburrida, no de no hacer nada, sino de estar en la casa. (Gleydi)

Yo soy una persona que le gusta estar sola, entonces tomarme mi tiempo para leer tranquila, para distraerme después de trabajar todo el día en la computadora. O escuchar música. O tomarme mi tiempo en silencio para descansar la cabeza. No tener ese tiempo y además viviendo en un departamentito, en un dos ambientes ni patio ni balcón. Entonces estar 24/7 encerrades. (Agustina)

No tenía tiempo ni concentración para estudiar. Ya cuando llegaba a mi casa solo quería dormir. (...) Sí, había veces que no dormía por tres días, y peor cuando me internaron a mi sobrina, no podía dormir nada. Me estaba haciendo mal eso. (Viviana)

Al preguntarles si habían podido compartir con otras personas estos sentimientos, para conocer si hubo una tramitación individual o colectiva, las cuatro jóvenes expresan diversas situaciones sobre la base de las relaciones interpersonales que habían construido y sus ámbitos de socialización, como vimos al inicio de este apartado. Viviana hizo referencia a que lo pudo hablar con sus amigos: «Sí lo pude hablar, más con mis amigos que otra cosa, que ellos siempre estaban ahí para darme una mano». Al igual que Agustina, quien enfatizó en que la acompañaron, más allá de que no tuvieron responsabilidades de cuidado 
infantil como ella: «Que más allá de que solo una tiene hije y es más grande que el mío. Más allá de eso, me súper entendieron, me súper apoyaron». Diferente fue el espacio construido por Valentina, donde la experiencia de cuidar y, particularmente, de maternar resultó estructurante de la creación de diálogo: «Más que nada poder hablarlo con amigas que quizás estén en la misma situación; mi hermana, por ejemplo, vive sola con su hija».

El núcleo de personas con las cuales compartieron sus sentimientos y generaron un espacio de diálogo (virtual mayormente), a partir del cual pudieron tramitar colectivamente las emociones, estuvo conformado mayormente por otras jóvenes. Se sabe que el grupo de pares es un espacio privilegiado de socialización y esto surge desde el reconocimiento de las vivencias compartidas con los amigos y las amigas que, muchas veces, devienen en sentidos compartidos y diferenciaciones con otros. En estos casos, especialmente del mundo adulto, ninguna de las jóvenes compartió sus sentimientos con su padre, madre, abuelo o abuela. Lo que puede habilitar la comprensión de esta experiencia en clave generacional. Una situación diferente se nos presenta con el testimonio de Gleydi, quien afirmó: «Específicamente de mis sentimientos no hablé con nadie, porque soy muy cerrada, no suelo compartir mis sentimientos con nadie, ni a mi esposo, muy rara vez le comento mis cosas». Antes de la pandemia, solamente concurría a su institución educativa y a la iglesia, sin hacer referencia a otros espacios de socialización juveniles ni a actividades con otras/os jóvenes, lo que creemos puede explicar esta soledad a la hora de poner en palabras y gestionar sus emociones. Tal como afirman Waisgrais y Aullicino (2018), el bienestar de quienes llevan adelante las tareas de cuidado afecta significativamente la vida de las infancias; por ello, sería interesante profundizar, en otro estudio, en el análisis en clave relacional de esta afectación. 


\section{CONSIDERACIONES FINALES}

En los últimos quince años, la Argentina ha tenido importantes avances en la ampliación de derechos de las mujeres y diversidades en pos de alcanzar la igualdad de género. No obstante estos adelantos, aún persisten brechas de género y muchas de ellas están siendo puestas en la agenda pública, como es el caso de la demanda de la necesidad de reconocer, redistribuir y reducir la carga de trabajo no remunerado que se desarrolla en la esfera familiar. En el contexto particular de la pandemia por Covid-19, las medidas de aislamiento social adoptadas por el gobierno nacional en marzo de 2020 con el lema «quédate en casa»y —entre otras disposiciones - el cierre de los espacios de cuidado para la primera infancia y el paso a la modalidad virtual de la totalidad del sistema educativo dejaron al descubierto la crisis del cuidado latente. Se llegó a una familiarización extrema que, por la dinámica patriarcal de nuestra sociedad, recayó en las mujeres e identidades feminizadas principalmente.

A lo largo del artículo, nos preguntamos cómo fueron las experiencias subjetivas de las jóvenes que desarrollaron tareas de cuidado infantil durante el aislamiento en la provincia de Mendoza, en pos de analizar las singularidades de un problema de carácter estructural como son las desigualdades de género y la crisis social, económica y sanitaria potenciada por la pandemia. Las jóvenes llevaron a cabo tareas de cuidado directo e indirecto, de manera no remunerada, a niños/as en sus hogares (tres tenían hijos/as y en un caso eran sus sobrinos/as), a quienes tuvieron que dedicar más tiempo que antes. En algunos casos, las mismas eran consideradas como responsabilidad de ellas, entonces tuvieron que gestionar la carga mental que implica cuidar; en otros casos, esa carga era depositada en sus madres. Sin embargo, no encontramos en sus discursos problematización alguna en torno a la organización política y social del cuidado ni a los roles socialmente construidos de las mujeres como cuidadoras; lo que aparentemente no dejaría lugar para poner en tensión lo posible y lo ideal en torno a dicho trabajo. Nos queremos detener en el testimonio de una de las jóvenes, 
quien es la única adulta de su hogar, en el que convive con su hijo de cinco años; es activista feminista, cuenta con el apoyo de su abuela para el cuidado y reflexiona: «Mi abuela me ayuda bastante con él (...). Pero bueno, siempre recae el trabajo sobre las mujeres y mi abuela ya crio mucho, entonces trato siempre de no llevarle tantas cosas». A través de este testimonio y de otras afirmaciones en las cuales reconoce los mandatos de género, vemos cómo es la única de las jóvenes que presenta una mirada crítica sobre las expectativas de género que recaen sobre ellas.

En un contexto como el argentino, donde a partir del 2015 crecieron los estudios sobre el involucramiento de las jóvenes en las demandas de los feminismos - lo que se conoce como «la revolución de las pibas»-, encontrarnos con estos testimonios nos ayuda a comprender las complejidades y las heterogeneidades dentro de aquello que nombramos como juventud. A partir de aquí nos surgen algunas preguntas para seguir trabajando: ¿hay una puesta en tensión de los mandatos de género en torno al cuidado por las jóvenes? ¿De qué maneras se pueden manifestar las tensiones entre lo posible y lo ideal? ¿La manifestación de cansancio, agobio y estrés por la intensificación de los cuidados puede llegar a ser una puerta de entrada para cuestionar los mandatos? ¿Qué rol ocupa el Estado en su visibilización?

El trabajo de cuidado que llevaron adelante tuvo un impacto en su salud mental, sus relaciones, sus trayectorias educativas y en su libertad económica (Young Women's Trust, 2020) y puso en valor la importancia de contar con un espacio personal, necesario para su bienestar integral. Ese «cuarto propio» que tenían en la prepandemia y que habían logrado complementar con las tareas de cuidado fue clausurado. Poniendo en tensión la obligación y el mandato de cuidar con sus deseos de tener tiempos individuales. Sin embargo, las experiencias son diferentes. Podemos reconocer cómo contar con diferentes capitales sociales, económicos y simbólicos permite manejar la tensión entre ideal (o deseo) y posibilidad de manera diferencial, amortiguando (o no) las consecuencias que las 
desigualdades de género provocan en las trayectorias de vida de las mujeres jóvenes.

Consideramos que el cuidado constituye uno de los nudos donde mejor se observan las inequidades de género, por ello esperamos que este artículo ayude a conocer los procesos subjetivos detrás de los números que nos arroja la pandemia en Argentina, a la luz de las particularidades de las vivencias de las jóvenes de Mendoza, para evitar caer en afirmaciones homogéneas y para gestionar políticas públicas integrales.

RECIBIDO: 30 DE JUNIO DE 2021 ACEPTADO: 12 DE AGOSTO DE 2021

\section{BIBLIOGRAFÍA}

Ahmed, S. (2008). The politics of good feeling. ACRAWSA, 4(1), 1-18. Recuperado https://static1.squarespace.com/static/58ad660603596eec00ce71a3/t/58b ecb77893fc0f $72747 \mathrm{~d} 4 \mathrm{e}$ 8/1488898936274/The+Politics+of+Good+Feeling.pdf

ANGilletta, F. (2021). Zona de promesas: cinco discusiones fundamentales entre los feminismos y la politica. Buenos Aires: Capital Intelectual.

ARZA, C. (2020). Familias, cuidado y desigualdad. Documentos de trabajo: Cuidados y mujeres en tiempos de COVID. Recuperado de: https://repositorio.cepal.org/bitstream/handle/11362/46453/S2000784_e s.pdf? sequence $=1$ \&isAl lowed $=y$

BALBo L. (1978). La doppia presenza. Inchiesta, VIII(32), 3-6.

Batthyany, K. (2013). Perspectivas actuales y desafíos del Sistema de Cuidados en Uruguay. En L. PAUTASSI y C. ZIBECCHI (coords.), Las fronteras del Cuidado, agenda, derechos e infraestructura. Buenos Aires: Biblos.

Bourdieu, P. (1997). Capital Cultural, Escuela y Espacio Social. Buenos Aires: Siglo XXI.

CABrera, M. y Ferreira, M. C. (2015). Afectos, emociones y sentimientos: reconfiguraciones de los órdenes de familia, parentesco, género y 
sexualidad. Presentación del dossier. Clínica \& Cultura, 4(1), 29-33. https://seer.ufs.br/index.php/clinicaecultura/article/view/4067

Caetano, G. y De Armas, G. (2015, marzo 30). Pobreza y desigualdad en América Latina (1980-2014). El País. http://www.pensamientocritico.org/gercae0415.pdf

CARMona, R. (2015). Repensar el rol del Estado argentino en clave latinoamericana. Avances y asignaturas pendientes durante la última época. Márgenes, revista de economía política, (1), 167-183.

CAstel, R. (1997). La metamorfosis de la cuestión social. Una crónica del salariado. Buenos Aires: Paidós.

Chaves, M. (2005). Juventud negada y negativizada: Representaciones y formaciones discursivas vigentes en la Argentina contemporánea. Última Década, 13(23), 9-32. https://dx.doi.org/10.4067/S071822362005000200002

Chaves, M., Cortés, F., Flaster, G., Galimberti, C. y Speroni, M. (2013). En busca de nuevas cartografías para un campo de estudios en consolidación: balance y perspectivas a seis años del informe «Investigaciones sobre juventudes en Argentina: estado del arte en ciencias sociales 1983-2006». Sudamericana: Revista de Ciencias Sociales, 2(2), 37-61. https://fh.mdp.edu.ar/revistas/index.php/sudamerica/article/view/857

COMisión ECONÓMICA PARA AMÉRICA LATINA Y EL CARIBE (CEPAL) (2020). Documentos de trabajo: Cuidados y mujeres en tiempos de COVID-19: la experiencia en la Argentina. CEPAL. https://repositorio.cepal.org/bitstream/handle/11362/46453/S2000784_e s.pdf? sequence $=1 \&$ isAl lowed $=\mathrm{y}$

DAICH, D. (2017). Aguafiestas porteñas. Sexo y dinero en la micropolítica emocional abolicionista. Cuadernos Pagu, 1(51), 1-32. https://doi.org/10.1590/18094449201700510008

Daich, D., Pita, M. V. y Sirimarco, M. (2007). Configuración de territorios de violencia y control policial: corporalidades, emociones y relaciones sociales. Cuadernos de Antropología Social, (25), 71-89. http://revistascientificas.filo.uba.ar/index.php/CAS/article/view/4379

DE LEÓN, G. (2017, marzo). Documento de Trabajo n¹58: Jóvenes que cuidan: impactos en su inclusión social. CIPPEC. Recuperado de: https:/www.cippec.org/wp-content/uploads/2017/04/158-DT-PSJovenes-que-cuidan-Gimena-d e-Leon-2017.pdf 
Díaz Lagou, G. y Della PaOlera, C. (2021, marzo). ¿Qué hay detrás de los números de pobreza de 2020? CIPPEC. Recuperado de: https:/www.cippec.org/textual/que-hay-detras-de-los-numeros-depobreza-de-2020/

ELIZALDE, S. (comp.) (2011). Jóvenes en cuestión: configuraciones de género y sexualidad en la cultura. Buenos Aires: Biblos.

FAUR, E. (2014). El cuidado infantil en el siglo XXI: Mujeres malabaristas en una sociedad desigual. Buenos Aires: Siglo Veintiuno Editores.

. (2009). Organización social del cuidado infantil en Ciudad de Buenos Aires. El rol de las instituciones públicas y privadas, 2005-2008. Tesis doctoral. Flacso-Argentina.

Faur, E. y Pereyra, F. (2018). Gramáticas del cuidado. En J. I. Piovanni y A. SALVIA (comps.), La Argentina en el siglo XXI: cómo somos, vivimos y convivimos en una sociedad desigual: Encuesta nacional sobre la estructura social (pp. 497-534). Buenos Aires: Siglo XXI.

FÉLIZ, M. (2015). Argentina neodesarrollista: Debates sobre el modelo. La Plata: EDULP.

Grupo de las Naciones Unidas para el Desarrollo Sostenible (2020). Jóvenes ante el Covid-19 en América Latina y el Caribe. CEPAL. Recuperado

de: https://www.cepal.org/sites/default/files/presentations/ppt_dia_internaci onal_de_la_juventu d_2 020.pdf

INDEC (2020). Mercado de trabajo. Tasas e indicadores socioeconómicos (EPH). Segundo trimestre de 2020. 4(5). Recuperado de: https://www.indec.gob.ar/uploads/informesdeprensa/mercado_trabajo_e ph_2trim20929E51916 1.pdf.

. (2015). Encuesta Nacional de Jóvenes 2014. Ministerio de Economía de la Nación, Presidencia de la Nación Argentina. Recuperado de: https://www.indec.gob.ar/ftp/cuadros/poblacion/resultados_enj_2014.pd f

Johnson, M. C., Saletti-Cuesta, L. y Tumas, N. (2020). Emociones, preocupaciones y reflexiones frente a la pandemia del COVID-19 en Argentina. Ciência \& Saúde Coletiva, 25(1), 2447-2456. https://doi.org/10.1590/1413-81232020256.1.10472020

Katzkowicz, S., La Buonora, L., Pieri, D., Pandolfi, J., Semblat, F., NuñEZ, S., SAUVAL, M. y ThEVENET, N. (2015). Cuadernos temáticos de la ENAJ: El trabajo de cuidados desde una perspectiva de género y 
generaciones. Unidad de Estudios del Instituto Nacional de la Juventud. MIDES. Recuperado de: https://medios.presidencia.gub.uy/tav_portal/2015/noticias/NO_R197/cu aderno-ENAJ.pdf

Lutz, C. y White, G. (1986). The anthropology of emotions. Annual Review of Anthropology, (5), 405-436. https://doi.org/10.1146/annurev.an.15.100186.002201

Manzanelli, P. y SchorR, M. (2013). Dinámica externa de la economía argentina. Un análisis a partir de las grandes firmas. En M. SCHORR (comp.), Argentina en la posconvertibilidad: ¿desarrollo o crecimiento industrial? Estudios de economía política (pp. 19-53). Buenos Aires: Miño y Dávila.

Ministerio De las Mujeres, GÉNERo y DiverSidAD (2020). Segundo encuentro de la mesa interministerial de Políticas de cuidado. Recuperado de: https://www.argentina.gob.ar/sites/default/files/politicascuidadocovid19. pdf

NuÑEZ, P. y LiTICHEVER, L. (2016). Radiografias de la experiencia escolar. Ser joven(es) en la escuela. Buenos Aires: Grupo Editor Universitario y CLACSO.

ORganización INTERNACIONAL DEL TRABAJO (OIT).(2020). Los jóvenes y la covid-19: efectos en el empleo, la educación, los derechos y el bienestar mental. OIT. Recuperado de: https://www.ilo.org/wcmsp5/groups/public/ed_emp/documents/publicati on/wcms_753054.pdf

PaUtassi, L. (2019). La emergencia del cuidado en los ámbitos locales. Múltiples configuraciones. En L. PAUTASSI (dir.), La agenda emergente de las políticas sociales: movilidad urbana, cuidado y violencias de género. Buenos Aires: Biblos.

Pérez, P. E. y Busso, M. (2018). Juventudes, educación y trabajo. En J. I. PIOVAnNi y A. SAlvia (comps.), La Argentina en el siglo XXI: Cómo somos, vivimos y convivimos en una sociedad desigual: Encuesta nacional sobre la estructura social (pp. 569-624). Buenos Aires: Siglo XXI.

Perez, L., Perez, R. y SecA, M.V. (2020). Metodología de la investigación cientifica. Buenos Aires: Maipue.

RaZAVI, S. (2007). The Political and Social Economy of care in a Development Context. Ginebra: UNRISD. 
RODRÍGUEZ EnRÍQUEZ, C. (2018). Economía del cuidado y desigualdad en América Latina: Avances recientes y desafíos pendientes. En C. Carrasco Bengoa (comp.), Economía feminista: desafios, propuestas y alianzas. Buenos Aires: Madreselva.

. (2014). El trabajo de cuidado no remunerado en Argentina: un análisis desde la evidencia del Módulo de Trabajo no Remunerado. Documentos de Trabajo Políticas públicas y derecho al cuidado, 2(2-2014), 1-24. $\quad$ http://www.ela.org.ar/a2/index.cfm?muestra \&aplicacion $=$ APP $187 \& \mathrm{cnl}=87 \&$ opc $=53 \&$ codconte nido $=2077 \&$ plcontampl $=4$

. (2005). La economía del cuidado: un aporte conceptual para el estudio de politicas públicas. Buenos Aires: CIEPP.

Rodríguez Enríquez, C., Alonso, V. y Marzonetto, G. (2020, abril 23). En tiempos de coronavirus, el trabajo de cuidado no hace cuarentena. Pensar la Pandemia. Observatorio Social de Coronavirus, 3. https://www.clacso.org/en-tiempos-de-coronavirus-el-trabajo-decuidado-no-hace-cuarentena/

SAlviA, A. (comp.) (2008). Jóvenes promesas. Trabajo, educación y exclusión social de jóvenes pobres en la Argentina. Buenos Aires: Miño y Dávila.

TESCH, R. (1990). Qualitative research: analysis types and software tools. Nueva York: The Falmer Press.

UNICEF (2020). Encuesta Covid: Percepción y actitudes de la población. Impacto de la pandemia y las medidas adoptadas sobre la vida cotidiana. Recuperado de: https://www.unicef.org/argentina/media/8061/file

Vega Solis, C. (2009). Culturas del Cuidado en transición: espacios, sujetos e imaginarios en una sociedad de migración. Barcelona: UOC (Universitat Oberta de Catalunya).

Vommaro, P. (2015). Juventudes y políticas en la Argentina y en América Latina. Buenos Aires: Grupo Editor Universitario.

WAISGRAis, S. y AUllicino, C. (2018). El cuidado y la primera infancia. En Las políticas de cuidado en Argentina: avances y desafios. PNUD, CIPPEC, Unicef y OIT.

YOUNG WOMEN's TRUST (2020). Valuing young women's unpaid work. London Young Researchers project. Recuperado de: https:/www.youngwomenstrust.org/wpcontent/uploads/2020/11/Valuing-young-womens-unpaid-work.pdf 